\title{
Thermal Study of HOM Couplers for Superconducting RF Cavities
}

\author{
M.Fouaidy, T Junquera \\ Institut de Physique Nucléaire (CNRS-IN2P3). 91406 ORSAY France \\ S.Chel, A.Mosnier \\ DSM/DAPNIA/SEA CE-Saclay 91191 GIF-SUR-YVETTE France
}

\begin{abstract}
The thermal behaviour of a "fish hook" type HOM coupler [1-2] for Superconducting RF Cavities is analyzed by numerical simulation using a 3D finite element based code for both $\mathrm{CW}$ (rings, recirculating linacs) and pulsed (s.c. colliders studies like TESLA) accelerator types. Consequently, a thorough study of the thermal stability of this system is necessary in both transient and steady-state regimes. Numerical simulations, assuming anomalous losses at the end of the HOM inner conductor, were performed. The effects of the HOM coupler geometry and the materials thermal conductivity on the critical heat flux inducing the quench of the system are discussed.
\end{abstract}

\section{INTRODUCTION}

The superconducting Higher Order Modes (HOM) couplers used for Superconducting Radiofrequency Cavities (SRF) cooled by Liquid Helium (LHe) are generally limited by thermal breakdown induced by various phenomena for accelerating fields $E_{\mathrm{acc}}$ in the range $2-5 \mathrm{MeV} / \mathrm{m}$ when no efficient cooling of the inner conductor is provided. These $E_{a c c}$ values are much smaller than the values to be reached for future projects (e.g. TESLA [3] : $\mathrm{E}_{\mathrm{acc}}=25 \mathrm{MeV} / \mathrm{m}$ ). We consider that HOM and beam tubes are located inside the insulation vacuum of the $\mathrm{LHe}$ vessel ; this indirect $\mathrm{LHe}$ cooling should obviously reduce the thermal quench limit of the HOM couplers. It is then necessary to choose properly the HOM coupler geometry and its construction material in order to increase its capability to whistand anomalous RF losses.

At low temperature, the RF surface resistance, the thermal conductivity $(k)$, the specific heat per unit volum $\left(C_{v}\right)$, the heat transfer coefficient at LHe-metal interfaces are strongly dependent on temperature $(T)$. The resulting heat equation with the pertinent boundary and initial conditions is then nonlinear. Moreover, the system studied is not axisymetrical, hence a 3D computer code is necessary. We used the CASTEM 2000 code [4] for this purpose : this Finite Element Method (FEM) based code can handle such problems in both transient and steady-state conditions for arbitrarly shaped computational domains with multiple regions (i.e. materials).

\section{METHOD AND ANALYSIS \\ A. The boundary value problem}

The temperature field $T(x, y, z, t)$, where $x, y, z$ and $t$ are spatial-coordinates and time respectively, within the computational domain $\Omega$ is governed by the well-known non-linear 3D Heat Diffusion equation with no heat source :

$$
\operatorname{div}\left(\mathrm{k}_{\mathrm{i}}(\mathrm{T}) \nabla \mathrm{T}\right)=\mathrm{C}_{\mathrm{v}_{\mathrm{i}}} \frac{\partial \mathrm{T}}{\partial \mathrm{t}}
$$

where the subscript " $i$ " refers to thermal properties within the region $\Omega_{\mathbf{i}}$.

For the cases of our concern, the typical boundary conditions are : prescribed heat flux $q$, heat transfer to the LHe at $T_{\text {bath }}$ controlled by the Kapitza conductance $\mathrm{H}_{\mathrm{K}}$ ( $\mathrm{T}_{\text {bath }}<$ $\mathrm{T}_{\lambda}=2.176 \mathrm{~K}$ ). For this parameter, we have used the following expression [5] :

$$
\mathrm{H}_{\mathrm{K}}=0.017 \mathrm{~T}_{\text {bath }}^{3.62}\left(1+\frac{3}{2}\left(\frac{\Delta \mathrm{T}}{\mathrm{T}_{\text {beth }}}\right)+\left(\frac{\Delta \mathrm{T}}{\mathrm{T}_{\text {bath }}}\right)^{2}+\frac{1}{4}\left(\frac{\Delta \mathrm{T}}{\mathrm{T}_{\text {beth }}}\right)^{3}\right)
$$

where $H_{K}$ unit is $W / \mathrm{cm}^{2} / K, \Delta T=T-T_{\text {bath }}$ and $T$ is the LHe-cooled surface temperature.

Concerning the transient computations, the whole system studied was assumed to be initially at the LHe temperature.

\section{B. Variational problem and approximation}

Using the so-called Galerkin Method, the semidiscrete problems corresponding to the above boundary value problem are derived. Briefly, this method constists on subdividing $\Omega$ into a set of subvolumes or finite elements, and expanding the solution in terms of FEM using $N$ components global basis functions. Then, a variational method is applied to these functions leading to $\mathrm{N}$ differential (algebraic) equations for time-dependant (steadystate) problems respectively. These equations constitute the semidiscrete variational problem, where the unknown are precisely the nodal values $\mathrm{Tj}(\mathrm{t})$ of the temperature field within the $\Omega$-mesh.

\section{Resolution Method}

In the steady-state case, the above non-linear system is solved by an iterative method using linearisation technique and a relaxation factor for improving the convergence of the process. The steady-state solution is reached when the relative variations of temperature at all the nodes of the $\Omega$-mesh is less than $10^{-3}$.

For the transient case, the above equations, discretized in time are solved by a n-step method : the temperature field at a given time is calculated from temperature fields at the $n$ preceeding times (we chose $n=2$ ). 


\section{APPLICATION TO THE HOM COUPLER}

\section{A. The model and thermal stability criteria}

The real system studied (Fig. 1) consists in the beam tube of the cavity, the HOM coupler (diam. : $40 \mathrm{~mm}$ and Inner Conductor (IC) diam. : $8 \mathrm{~mm}$ ), the $\mathrm{Nb}$ flange of cryostat tank and the LHe cooled part of the cavity. For numerical simulation, this system was modelled using a slighty modified geometry (Fig. 2) which includes all the parts described previously up to the first iris of the cavity. Notice however that the thermal contact resistances at the sealings (i.e. flanges of the HOM) were neglected, the fishhook has been replaced by a straight Inner Conductor (IC) which has no influence on the numerical results because the thermal radiations are negligible $(T<10 \mathrm{~K})$, and we have also neglected the heat flux coming from the RF coaxial cable (using a simple $\mathrm{Nb}$ cover in place of the upper part of the HOM).

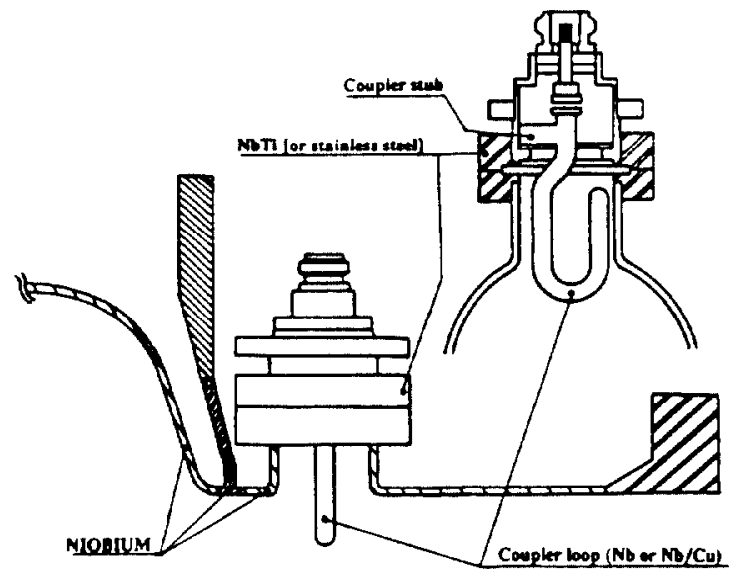

Fig. 1 : Cavity-HOM design.



Fig. 2 : Meshed cavity-HOM assembly and applied boundary conditions.

In the defect free case, the total RF losses dissipated on the IC of the HOM remain lower than $0.4 \mathrm{~mW}$ (with a peak heat flux density of $40 \mu \mathrm{W} / \mathrm{cm}^{2}$ ) for $T_{\text {bath }}=2.0 \mathrm{~K}$,
$\mathrm{E}_{\mathrm{acc}}=25 \mathrm{MeV} / \mathrm{m}$ and a residual surface resistance $R_{\text {res }}=20 \mathrm{n} \Omega$ [6]. These very low RF dissipations can not explain an increase of the hot spot temperature beyond the $\mathrm{Nb}$ critical temperature $\mathrm{Tc}=9.2 \mathrm{~K}$. Consequently, anomalous RF dissipation sources (cavity electron loading, surface defect, multipactor, ...) have to be considered as possible causes of the HOM coupler thermal breakdown. We have then assumed a highly dissipative area, located at the end of the IC, the farest point from the cold source. With this pessimistic assumption we will obtain the minimum heat flux limit $Q_{c}$ before the quench.

The thermal stability will be insured if two conditions are fulfilled : the temperature $T_{\max }$ at the hot spot must be lower than $T_{c}=9.2 \mathrm{~K}$ and the maximum heat flux density transfered to the LHe bath must be below the critical heat flux inducing the Film-Boiling limit.

\section{$B$. Steady state regime}

The steady-state temperature distribution was computed up to the critical heat flux $\left(Q_{c}\right)$ for the following arrangements and the results are summarized in Table 1.:

\# 1. the whole HOM coupler-cavity assembly is machined from bulk Niobium with $R R R=40,194$ or 570 ,

\# 2. all these parts are in bulk Niobium (RRR = 40,194 or 570) excepted the IC and the Stub which are made of sputtered Niobium onto a Copper substrate of $R R R=300$,

\# 3. the same material as in item\#1 but with addition of a copper $(\mathrm{Cu}: \mathrm{RRR}=300)$ thermal shunt between the Coupler and the LHe cryostat flange (see Fig. 2),

\# 4 the same arrangement as in item \#2 with addition of the thermal shunt described in item \#3.

\begin{tabular}{||l|c|c|}
\hline Nb quality & Arrangement & Critical heat flux $\mathrm{Q}_{\mathrm{c}}(\mathrm{mW})$ \\
\hline \hline RRR 40 & $\# 1$ & 72 \\
\hline RRR 194 & $\# 1$ & 314 \\
\hline RRR 570 & $\# 1$ & 925 \\
\hline \hline RRR 40 & $\# 2$ & 600 \\
\hline RRR 194 & $\# 2$ & 1860 \\
\hline RRR 570 & $\# 2$ & 3418 \\
\hline RRR 40 & $\# 3$ & 79 \\
\hline RRR 194 & $\# 3$ & 347 \\
\hline RRR 570 & $\# 3$ & 1017 \\
\hline \hline RRR 40 & $\# 4$ & $2700^{*}$ \\
\hline RRR 194 & $\# 4$ & $3328^{*}$ \\
\hline RRR 570 & $\# 4$ & 5430 \\
\hline
\end{tabular}

Table 1 : Critical heat flux inducing the HOM coupler thermal breakdown in the steady-state regime.

* Quench limited by the transition to film boiling on the LHe-cooled $\mathrm{Nb}$ flange (heat flux density : $2.4 \mathrm{~W} / \mathrm{cm}^{2}$ ).

Analysing the temperature profiles along the IC for the arrangements \#1 and \#3 with $Q=Q_{c}$, we note that the non-linear effects increase with the Nb RRR, the temperature difference along the IC is around $3.3 \mathrm{~K}$ whatever the RRR may be, there is no sensible effect of the thermal shunt on $Q_{c}$ which increases (from nearly $70 \mathrm{~mW}$ to $1 \mathrm{~W}$ ) with the Nb RRR (see Table 1). The values of the 
thermal impedance of the IC $\left(R_{t h}=\Delta T_{I C} / Q_{c}\right)$ are $46 \mathrm{~K} / \mathrm{W}$, $10.5 \mathrm{~K} / \mathrm{W}$ and $3.6 \mathrm{~K} / \mathrm{W}$ for $\mathrm{RRR}=40,194$ and 570 respectively. So, even in the best arrangement (\#3 and $\mathrm{Nb}$ $\mathrm{RRR}=570$ ), the system is limited by the Niobium thermal conductivity.

In order to overcome this limitation, the arrangement \#2 was examined, leading to a reduction by a factor 6 of the IC thermal impedance. The adding of a thermal shunt (arrangement \#4) improves greatly $\mathrm{Q}_{\mathrm{c}}$ (Fig. 3) due to the sufficiently small thermal impedance $(0.6 \mathrm{~K} / \mathrm{W})$ of the IC, and in this computation, $70 \%$ of Qc is derived through the thermal shunt.



Fig. 3 : Temperature profile along the LHe cooled Nb flange (sketched in the insert).

Full line : with thermal shunt $\left(Q_{c}=5.4 \mathrm{~W}\right)$

Dashed line : without thermal shunt $\left(\mathrm{Q}_{\mathrm{c}}=3.4 \mathrm{~W}\right)$

\section{Transient regime}

This study was limited to the arrangement \#1 with $R R R=194$. The applied heat flux was pulsed close to the TESLA cycle (pulse length $\tau_{\mathrm{p}}=2 \mathrm{~ms} ; \mathrm{f}_{\mathrm{rep}}=10 \mathrm{~Hz}$ ). The maximum hot spot temperature during the RF pulse increases up to $8.6 \mathrm{~K}$ at the end of the first RF pulse for $Q_{c}=6 \mathrm{~W}$, does not recover its initial temperature (i.e. $\mathrm{T}_{\text {bath }}=1.8 \mathrm{~K}$ ) at the end of the first pulse $(4.4 \mathrm{~K})$, but reaches its stationnary value (9.2K) in nearly 10 pulses (Fig. 4). The higher temperature after an infinity of pulses as function of the heat flux can be evaluated with :

$$
\mathrm{T}_{\max } \cong 3.63 \mathrm{Q}^{0.35}+0.31 \mathrm{Q}^{0.49} \text {. }
$$

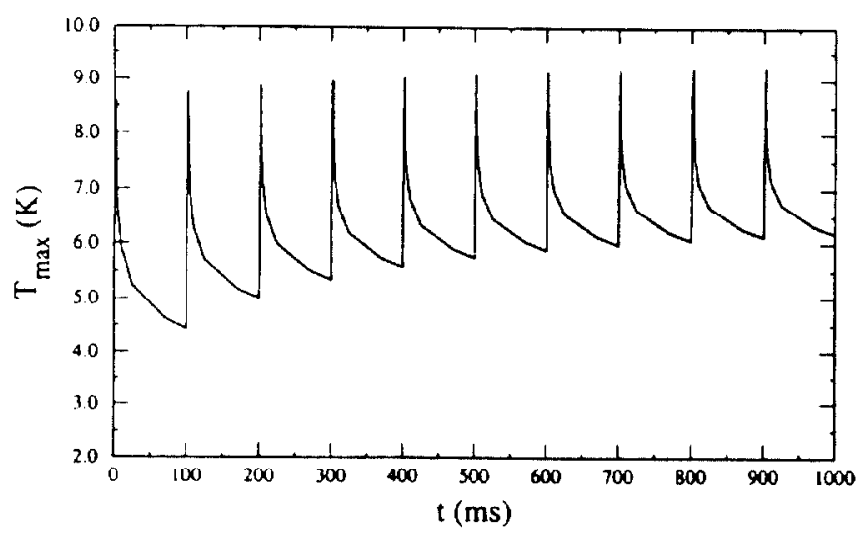

Fig. 4 : Temperature of the hot spot vs time
From the evolution of the temperature along the IC at different times (Fig. 5), the value of $300 \mathrm{~cm}^{2} / \mathrm{s}$ is obtained for the diffusion coefficient. Consequently, the perturbation during the RF pulse is localized close to the defect point (diffusion length of $1.5 \mathrm{~cm}$ in $2 \mathrm{~ms}$ ) and the corresponding heat flux can not be evacuated in one complete cycle $(110 \mathrm{~cm}$ in $100 \mathrm{~ms}$, that is to say, the length of the IC).

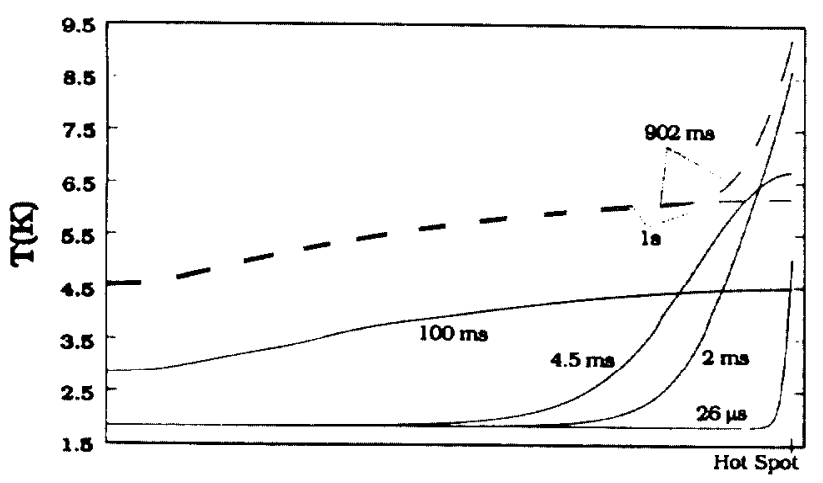

Fig. 5 : Temperature profile along the IC at different times.

Full lines : First pulse

Dashed lines : $10^{\text {th }}$ pulse

\section{CONCLUSION}

A HOM coupler heat load capability of over $5 \mathrm{~W}$ has been reached in case of a continuous anomalous RF dissipation, by using an IC and a stub made with sputtered $\mathrm{Nb}$ onto a $\mathrm{Cu}$ substrate, and adding a thermal shunt connected to the LHe flange. In the transient regime, the maximum heat load sustained by the bulk $\mathrm{Nb}$ coupler is $6 \mathrm{~W}$. In all the cases and for the corresponding critical heat fluxes, the maximum temperature at the iris of the cavity does not exceed $0.1 \mathrm{~K}$ with respect to $\mathrm{T}_{\mathrm{bath}}$, so that the thermal stability of the cavity is also insured.

We plane some thermal measurements on a special cavity/HOM assembly, to confirm these calculations and to take into account, if necessary, the effect of the contact resistances at the sealings.

\section{REFERENCES}

[1] A. Mosnier, "Developments of HOM couplers for superconducting cavities". 4th Workshop on RF Superconductivity, Tsukuba, 1989.

[2] Ph. Bernard and al., "Demountable E/H field HOM for the Nb-sputtered 4-cell LEP cavity". Sth Workshop on RF Superconductivity, Hambourg, 1991.

[3] H. Padamsee, "Review of the superconducting approach to linear colliders". Proc. 3rd Eur. Part. Conf., Berlin, 1992. [4] P. Verpeaux and al., "A Modern Approch of a Large Computer Code for Structural Analysis", Structural Mechanics Reactor, $n^{\circ} 10$, Los Angeles, 1989.

[5] K. Mittag, Cryogenics 13 (1973) 94.

[6] S. Chel, M. Fouaidy, "Thermal behavior of HOM couplers". Internal Report, CE-Saclay/DAPNIA/SEA-93. 